\title{
One Case of BRCA2 Germline Mutation Ovarian Cancer Mother and Carrier Daughter found by Genetic Counsel- ing
}

Eun Jung Lee', Hee Jeong Jeong ${ }^{1}$ and Min Kyu Kim²*

'Department of Obstetrics and Gynecology, Samsung Medical Center, Sungkyunkwan University School of Medicine, Seoul, Korea

2Department of Obstetrics and Gynecology, Samsung Changwon Hospital, Sungkyunkwan University School of Medicine, Changwon, Korea

\begin{abstract}
Among cause of carcinogenesis, heredity is believed to take about 10 percent in ovarian cancer. BRCA1 or BRCA2 account for largest portion of Hereditary Breast and Ovary Cancer (HBOC). Frequency of $B R C A 1 / 2$ germ line mutations varies according to region and ethnicity from 1.1-39.7 percent. The identification of ovarian cancers with a BRCA mutation is will be more and important due to the possibility to offer a genetic counseling and also due to potential beneficial treatment effects with a poly-ADP-ribose polymerase inhibitor in some individuals. We report the case of a 41 year old woman with a stage Ic mucinous ovarian adenocarcinoma and carrier daughter found on family genetic counseling. We indentified other family members with a history of breast cancer of 1 st degree and pancreatic cancer of 2 nd degree relative. After a screening with immunohistochemistry, the absence of nuclear expression for $B R C A 1$ and $B R C A 2$ was revealed. The gene sequencing confirmed heterozygous mutations of $B R C A 2$ gene. The daughter of the case subject consented for a test. This test was shown the daughter is positive for BRCA2 mutation. Regular surveillance, chemoprophylaxis with oral contraceptive and prophylactic surgery after childbearing were offered to her.
\end{abstract}

Key words: Ovarian carcinoma, BRCA mutation

\section{Introduction}

Among cause of carcinogenesis, heredity is believed to take about 10 percent in ovarian cancer. BRCA1 or BRCA2 account for largest portion of Hereditary Breast and Ovary Cancer (HBOC). ${ }^{1}$ Frequency of BRCA $1 / 2$ germ line mutations varies according to region and ethnicity from 1.1-39.7percent. ${ }^{2}$ Among korean ovarian cancer patients, they have been reported to be $2.7 \%(1 / 37)^{3)}$ and $23.8 \%$ $(15 / 63)^{4)}$ regardless of family history. It is hard to counsel patient's family and encourage genetic test for them. In addition, there is not much report about family counseling and penetrance report among ovary cancer patient in korea.

The average lifetime risk of breast or ovarian cancer in women with a BRCA 1 mutation is $65 \%$ and $39 \%$. The corresponding estimates for women with a BRCA2 mutation are $45 \%$ and $11 \%$, respectively. ${ }^{5)} B R C A 1 / 2$-associated ovarian carcinomas were associated with a longer overall survival time, ${ }_{1}^{6,7)}$ an increased sensitivity to platinum-based chemotherapy ${ }^{7,8)}$ and an increased sensitivity to a therapy with poly-ADP-ribose polymerase (PARP) inhibitors, ${ }^{9)}$ compared to their sporadic counterparts.

Received: 7 November 2013 Revised: 19 December 2013, Accepted: 20 December 2013, Published: 31 December 2013

${ }^{*}$ Corresponding author: Min Kyu Kim, M.D.

Division of Gynecologic oncology, Department of Obstetrics and Gynecology, Samsung Changwon Hospital, Sungkyunkwan University School of Medicine, 50 Hapsung-Dong, Masan Hoewon-Gu, Changwon-Si, Gyeongsangnam-Do 630-723, Korea

Tel: +82-55-290-6040, Fax: +82-2-6442-9285, E-mail: minkyukim@skku.edu

Conflict of interest: We declare that we do not have any conflicts of interests.

(c) This is an open-access article distributed under the terms of the Creative Commons Attribution Non-Commercial License (http://creativecommons.org/licenses/by-nc/3.0/) which permits unrestricted non-commercial use, distribution, and reproduction in any medium, provided the original work is properly cited.

(c) Copyright 2013 by the Korean Society of Medical Genetics 
Recognition of inherited risks allows a targeted prevention. The genetic $B R C A$ mutation testing is usually offered to women with a strong family history of breast and ovarian cancers. To estimate the risk for clinically-significant $B R C A$ mutations is the first step of the genetic test process. However to select suitable patients for a genetic testing in the clinic is not common yet.

Until today, ovarian cancer surveillance was not effective. Screening with CA 125 and ultrasound is not believed to reduce mortality. ${ }^{10)}$ However the risk of ovarian, fallopian tube and breast cancer can be reduced due to a risk-reducing salpingooophorectomy. ${ }^{11)}$

We report a case of a mother with an ovarian carcinoma and a daughter who was a BRCA2 mutation carrier which was identified by genetic counseling.

\section{Case}

A 41 year old woman G1P1 with findings concerning for an ovarian mass was referred from a local clinic to the Samsung Changwon Hospital. Due to a computed tomography (CT) scan of the abdomen and pelvis the presence of a 12-cm cystic and solid mass in the left ovary was confirmed No ascites or significant lymphadenopathy were detected (Fig. 1A). In the preoperative laboratory sample a slight elevation of CA-125 (37.71 U/mL) was shown.
After the workup, the surgery was laparoscopic performed. Mucinous borderline tumor of the left ovary with a frozen section diagnosis was reported during operation. But a reoperation for cancer staging was recommended because final pathology showed mucinous adenocarcinoma. The patient underwent a total laparoscopic hysterectomy, right salpingooophorectomy, pelvic lymph nodes dissection, para-aortic lymph nodes dissection and an infracolic omentectomy. The pathologic analysis by a gynecologic pathologist revealed no additional evidence of malignancy. The patient's final surgical staging was Ic. After the surgery, combination chemotherapy with six cycles of paclitaxol and carboplatin of 3-week intervals was given to the patient.

We found she had family members with a history of breast and pancreatic cancer (Fig. 1B). She consented to an IHC screening for the expression of BRCA1 and BRCA2. The IHC for BRCA1 and BRCA2 was negative (Fig. 1C, 1D). After obtaining an informed consent for the collection of blood samples, a genomic strand of DNA was extracted from peripheral blood and analyzed for mutations of the BRCA1 and BRCA2 genes. Following results were revealed: Nonsense mutation (Arg2494X) at the $7480^{\text {th }}$ base of Exon 15 in BRCA2 (Fig. 2).

Based on this genetic evaluation, we advised the patient to investigate her family members. Only her 23 year old daughter accepted for a test. This test was positive for the same BRCA2 mutation.
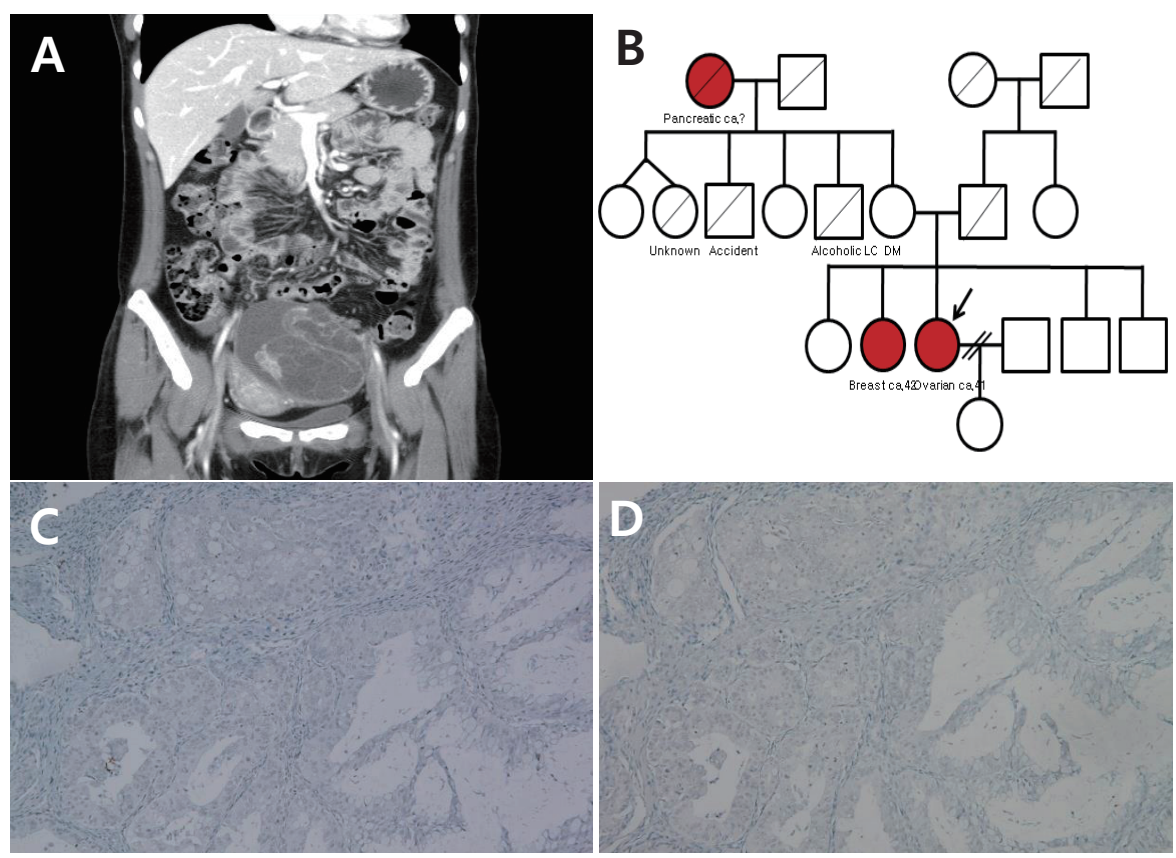

Fig. 1. A) Abdomen and Pelvis CT, B) Pedigree, C) BRCA1 immunohistochemistry (X200), D) BRCA2 immunohistochemistry (X200). 


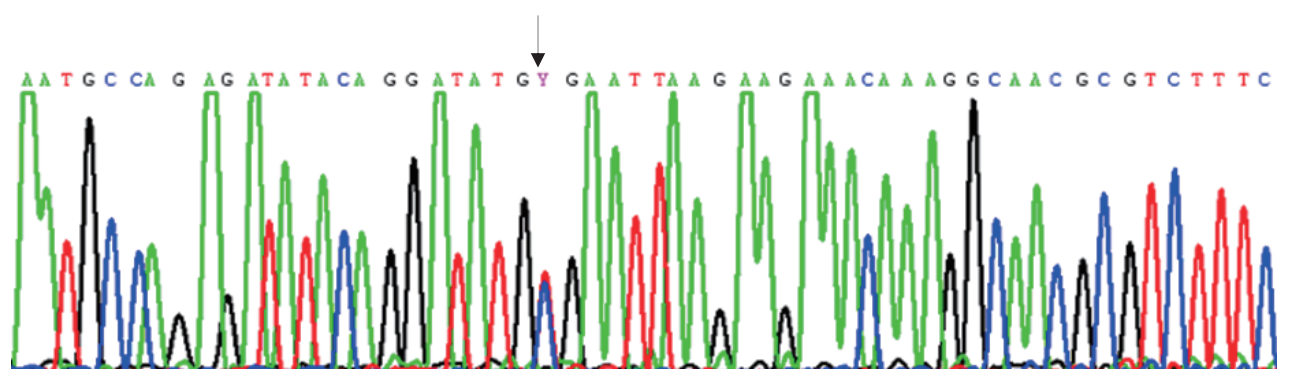

Fig. 2. BRCA2 gene sequencing result [Exon15, 7480 C>T (Arg2494X)].

\section{Discussion}

Prevention and early detection are key factors for an ovarian carcinoma risk reduction.

It is important to find the germ line mutations because they have a high risk of cancer penetrance. Otherwise we should be very careful to recommend an expensive genetic sequencing based on objective evidence. This is the first case of an ovarian carcinoma mother and a BRCA2 germ line mutation carrier daughter found by genetic counseling in Korea.

Genetic counseling for the patient and the daughter are different. A prophylactic surgery or close observation for breast cancer were offered to patient. A prophylactic mastectomy reduces the risk of developing breast cancer by more than 95\% and consequently reduces the breast cancer-specific mortality by $90 \% .{ }^{12)}$ A daughter was provided information with close survelliance, chemoprevention with oral contraceptive and risk reducing surgery after childbearing.

BRCA 1 mutations are found in $1.4 \%$ to $13.3 \%$ of sporadic ovarian cancers, and mutations of BRCA2 are identified in $0.9 \%$ to $8.0 \%$ of sporadic ovarian cancers. New advances in genomic technologies will likely accelerate the discovery of additional cancer susceptibility genes and increase the feasibility of comprehensive, simultaneous evaluation of multiple genes at low costs. Theoretically, most inherited risks for ovarian carcinoma could be identified before the onset of cancer if the recognition of genetic risks will improve. Also the proportion of ovarian carcinomas associated with inherited risk factors could be effectively prevented. The genetic of hereditary ovarian cancer is rapidly evolving, but $B R C A 1 / 2$ mutations remain the most common cause of the hereditary ovarian cancer.

Therefore, active family counseling can be a help in the diagnosis of $B R C A$ mutations and can also suggest the need for genetic sequencing in patients without a known family history of cancer.

\section{References}

1. Lynch HT, Casey MJ, Snyder CL, Bewtra C, Lynch JF, Butts M, et al. Hereditary ovarian carcinoma: heterogeneity, molecular genetics, pathology, and management. Mol Oncol 2009;3:97-137.

2. Shanmughapriya $S$, Nachiappan $V$, Natarajaseenivasan K. BRCA1 and BRCA2 mutations in the ovarian cancer population across race and ethnicity: special reference to Asia. Oncology 2013;84:226-32.

3. Kim YT, Nam EJ, Yoon BS, Kim SW, Kim SH, Kim JH, et al. Germline mutations of BRCA1 and BRCA2 in Korean sporadic ovarian carcinoma. Gynecol Oncol 2005;99:585-90.

4. Lim MC, Kang S, Seo SS, Kong SY, Lee BY, Lee SK, et al. BRCA1 and BRCA2 germline mutations in Korean ovarian cancer patients. J Cancer Res Clin Oncol 2009;135:1593-9.

5. Antoniou A, Pharoah PD, Narod S, Risch HA, Eyfjord JE, Hopper JL, et al. Average risks of breast and ovarian cancer associated with BRCA 1 or BRCA2 mutations detected in case Series unselected for family history: a combined analysis of 22 studies. Am J Hum Genet 2003;72:1117-30.

6. Chetrit A, Hirsh-Yechezkel G, Ben-David Y, Lubin F, Friedman E, Sadetzki S. Effect of BRCA1/2 mutations on long-term survival of patients with invasive ovarian cancer: the national Israeli study of ovarian cancer. J Clin Oncol 2008;26:20-5.

7. Yang D, Khan S, Sun Y, Hess K, Shmulevich I, Sood AK, et al. Association of BRCA1 and BRCA2 mutations with survival, chemotherapy sensitivity, and gene mutator phenotype in patients with ovarian cancer. JAMA 2011;306:1557-65.

8. Vencken PM, Kriege M, Hoogwerf D, Beugelink S, van der Burg ME, Hooning MJ, et al. Chemosensitivity and outcome of BRCA 1- and BRCA2-associated ovarian cancer patients after first-line chemotherapy compared with sporadic ovarian cancer patients. Ann Oncol 2011;22: 1346-52.

9. Fong PC, Boss DS, Yap TA, Tutt A, Wu P, Mergui-Roelvink M, et al. Inhibition of poly (ADP-ribose) polymerase in tumors from BRCA mutation carriers. N Engl J Med 2009;361:123-34.

10. Buys SS, Partridge $E$, Black $A$, et al. Effect of screening on ovarian cancer mortality: the Prostate, Lung, Colorectal and Ovarian (PLCO) Cancer Screening Randomized Controlled Trial. JAMA 2011;305:2295-303. 
11. Rebbeck TR, Kauff ND, Domchek SM. Meta-analysis of risk reduction estimates associated with risk-reducing salpingo-oophorectomy in BRCA1 or BRCA2 mutation carriers. J Natl Cancer Inst 2009;101:80-7.

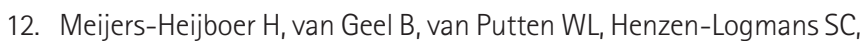

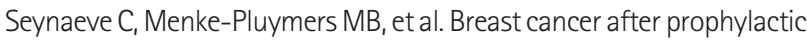
bilateral mastectomy in women with a BRCA1 or BRCA2 mutation. N Engl J Med 2001;345:159-64. 